\title{
Quel avenir pour l'Association africaine francophone de formation continue en hépato-gastroentérologie ?
}

\author{
Which future for the African Hepato-Gastroenterology French Speaking CME Association?
}

\author{
V. Lamy $\cdot$ C. Tzeuton $\cdot$ P. Couzigou \\ C) Springer-Verlag France 2012
}

\section{Scripta manent, verba volent ...}

Notre association a vu le jour il y a 15 ans à l'instigation de nos amis Paul Mainguet, Francis Klotz, Mohamed Fadel Ndiaye, Naïma Amrani, Fernand Vicari et quelques autres visionnaires qui se reconnaîtront mais que nous ne pouvons tous énumérer ici. Leur idée géniale et généreuse de départ était de réaliser un organe de formation médicale continue en hépato-gastroentérologie en Afrique pour les Africains et de démontrer son utilité.

L'historique de cette association a été retracé dans un excellent article du $J A H G$ (2007) 3-4:107-9

$\mathrm{http} / / /$ link.springer.com/article/10.1007/s12157-008-0033-1

Depuis sa création 15 réunions ou congrès ont eu lieu :

- Dakar (6-8/05/1998)

- Marrakech (17-18/04/1999)

- Yamoussoukro (27-29/04/2000)

- Rabat (1-3/11/2001)

- Tunis (2-4/05/2002)

- Dakar (27-29/11/2003)

- Yaoundé $(25-27 / 11 / 2004)$

- Alger (15-17/12/2005)

- Libreville (30/11-03/12/2006)

- Dakar (28/11-1/12/2007)

- Fès (9-11/10/2008)

- Yaoundé (22-24/10/2009)

\section{Lamy $(\bowtie)$}

Service d'hépato-gastroentérologie, $\mathrm{CHU}$ de Charleroi, Belgique

e-mail : vincent.lamy@chu-charleroi.be

\section{Tzeuton $(\triangle)$}

BP 12663, Douala, Cameroun

e-mail : tzeuton.christian@yahoo.fr

P. Couzigou $(\bowtie)$

Service d'hépato-gastroentérologie,

Hôpital du Haut-Levêque, CHU de Bordeaux,

33000 Bordeaux

e-mail : patrice.couzigou@chu-bordeaux.fr
- Tunis (27-29/05/2010)

- Brazzaville (10-12/11/2011)

- Alger (13-15/12/2012)

L'association a été enregistrée en France comme association sans but lucratif (loi 1901) le 28 octobre 1999.

Le conseil d'administration se réunit deux fois par an au moins durant les Journées francophones en France et durant les Journées africaines dans le pays organisateur. Une assemblée générale se déroule chaque année.

Plusieurs sociétés savantes d'Afrique francophone et d'Europe font à présent partie de l'association (voir « liens » sur notre site internet).

Un Journal africain de gastroentérologie et d'hépatologie $(J A H G)$ a vu le jour et se développe rapidement.

Un système de bourses pour permettre aux jeunes lauréats primés d'assister aux autres réunions de l'association a été lancé et connaît un vif succès.

Une première étude panafricaine sur l'hémorragie digestive a été rendue possible par un autre partenariat avec l'ANGH, la SNFGE et Astra Zeneca International et a permis de collecter des résultats très intéressants qui ont été présentés aux JFHOD (Journées Francophones d'Hépato-gastroentérologie et d'Oncologie Digestive) à Paris en 2011 et elle devrait être publiée :

http://www.snfge.asso.fr/01-bibliotheque/0a-resumes-jfpd/ 2011/5142.html

L'AAFFCHGE fait partie chaque année du « village francophone » aux cours des JFHOD à Paris et c'est l'occasion d'y présenter nos réalisations et futures réunions en Afrique :

http://www.jfhod.com/

Un site internet vient d'être lancé avec le soutien de notre association amie la FMC-HGE avec laquelle nous avons signé un accord de partenariat pour d'harmoniser nos actions de FMC :

www.aaffchge.org

Tous ces acquis sont autant de preuves tangibles de la nécessité d'avoir créé une telle association. 
Son succès peut se mesurer par l'assistance croissante aux congrès, le nombre de sociétés membres de l'association et la qualité des orateurs et des travaux présentés et publiés en quelques années.

Les JGAF (Journées de Gastroentérologie d'Afrique Francophone) sont devenues en 15 ans le rendez-vous des forces vives de l'hépato-gastroentérologie francophone africaine. Ces journées se tiennent souvent durant des journées nationales et permettent aux hépato-gastroentérologues, aux chirurgiens, aux radiologues digestifs et aux infirmières d'échanger, de se retrouver et d'apprendre dans une osmose stimulante.

A présent et dès lors qu'a été démontré l'utilité de cette entreprise, il convient aussi de faire le point sur son fonctionnement et d'envisager son avenir.

L'association vit malheureusement uniquement de bonnes volontés, du dynamisme de certains de ses membres et de ressources modestes générées par les cotisations de ses sociétés savantes membres et du partenariat de l'industrie.

L'association doit établir un plan d'action pour les années à venir pour assurer son indépendance financière, consolider ses alliances, trouver de nouveaux appuis, mieux se faire connaître et établir un organigramme de fonctionnement des structures.

\section{Assurer son indépendance financière}

Les sources de revenus de l'association sont très précaires; elles se répartissent en :

- Cotisations ou contributions annuelles versées par les sociétés savantes nationales, membres de l'association. Ces cotisations sont fonction du pays membre et du nombre de membres.

- Partenariat, essentiellement les contributions de nos partenaires pour la prise en charge des congressistes et des orateurs et l'obtention de bourses.

- Bénéfices des réunions et congrès permettant :

- aux organisateurs locaux de récupérer les mises de fonds consenties,

- à l'association de fonctionner et si possible d'accumuler quelques réserves,

- ces réserves sont essentiellement utilisées pour la prise en charge et les bourses octroyées aux jeunes collègues les plus méritants.

Le bilan financier de l'association doit être transparent. Un compte-rendu annuel avec aperçu clair des recettes et dépenses est indispensable. Une prévision budgétaire sur base annuelle et si possible pluriannuelle est nécessaire.

Les bénéfices des congrès devraient être aussi clairement connus et répartis en trois :
- un tiers pour la société nationale organisatrice,

- un tiers pour le fonctionnement propre de l'association,

- un dernier tiers pour la perpétuation et la structuration des congrès à venir.

Le partenariat de l'industrie (pharmaceutique et/ou endoscopique) est aléatoire, inconstant et en nette régression depuis quelques années. Il est donc urgent d'essayer d'obtenir un financement complémentaire alternatif et durable d'autres origines.

\section{Consolider les alliances}

Notre association est une entreprise remarquable de collaboration internationale scientifique, culturelle et humaine entre des sociétés savantes de deux continents. C'est un lien majeur entre les hépato-gastroentérologues francophones du Nord et du Sud de l'Afrique et d'Europe.

\section{Ouvertures africaines}

\section{Les sociétés membres}

Elles sont au nombre de 12 avec 18 pays représentés. Il faut encore accueillir d'autres pays africains.

\section{L'AMAGE (African and Middle East Association of Gastroenterology)}

Organe de l'OMGE/WGO (Organisation Mondiale de Gastroentérologie) pour représenter l'Afrique et le MoyenOrient en tant que $4^{\mathrm{e}}$ région. Plusieurs de nos représentants y sont actifs dont notre président qui y est vice-président :

http://www.worldgastroenterology.org/wgo-membershipafrica-mid-east.html

\section{Le centre de formation post-graduée en gastroentérologie de l'OMGE}

Ouvert à Rabat grâce au dynamisme de notre amie Naïma Amrani a déjà organisé de nombreuses sessions de cours : http://wgo-rtc.um5s.ac.ma/

\section{Ouvertures sur le monde}

\section{En Europe}

Nos paretenaires naturels sont les sociétés savantes nationales francophones. Des sociétés non francophones mais francophiles pourraient nous rejoindre à travers leurs membres francophones (Italie, Espagne, Allemagne, Suisse, Roumanie, etc). 


\section{En Amérique du nord}

Les premiers contacts avec nos collègues de l'association des gastroentérologues du Québec avaient été prometteurs mais sans lendemain. Ceux-ci devraient être relancés pour aboutir sur des participations à nos activités et des contacts avec les universités et les organismes canadiens et internationaux implantés au Canada (Agence de la Francophonie, Aupelf-Uref).

\section{En Asie}

Une association sœur de FMC en HGE de pays francophones ou partiellement francophones d'Asie existe et se réunit tous les deux ans à Hanoi au Vietnam. C'est d'ailleurs elle qui avait inspiré un de nos fondateurs. Nous pourrions les inviter et les informer de nos activités.

\section{Dans l'Océan indien}

Des contacts avec Maurice, Madagascar et la Réunion devraient être rétablis.

\section{Dans les Caraïbes}

De même des contacts avec les départements français devraient être possibles.

\section{Trouver de nouveaux appuis}

\section{L'industrie pharmaceutique}

Elle représente et doit rester un partenaire privilégié. Nous devons trouver les interlocuteurs responsables et essayer à travers notre association - et son éventuel comité d'éthique - d'avoir la possibilité de réaliser des études cliniques multicentriques nationales ou internationales.

\section{Les sociétés de matériel endoscopique}

Elles doivent également rester des partenaires. Des contacts ont déjà permis la réalisation d'ateliers de formation en endoscopie digestive.

\section{Les sociétés d'imagerie}

Celles-ci commencent aussi à être intéressées par nos activités. L'échographie abdominale - directement accessible aux gastroentérologues - constitue une activité importante et un marché potentiel pour ces sociétés.
Les organismes de coopération bilatérale

Ils devraient être mieux sensibilisés à nos activités.

\section{Les organismes internationaux}

\section{L'Agence de la Francophonie}

Est informée de nos activités en la personne de son Secrétaire Général, le Président Abdou Diouf mais reste trop frileuse pour une aide structurelle. Elle nous a accordé une année quatre bourses :

http://www.auf.org/

\section{La Conférence Internationale des Doyens et des Facultés et Ecoles de Médecine d'expression française (CIDMEF)}

Le site :

http://www.cidmef.u-bordeaux2.fr/

Conférence visitée par le Professeur Couzigou qui y a présenté, à Libreville en 2011, la structure de notre association : http://www.canal-u.tv/video/canal_u_medecine/cidmef libreville_2011_les_objectifs_de_la_cidmef.7152

\section{L'Aupelf-Uref}

Association d'universités francophones ou partiellement francophones qui devrait bénéficier de notre réseau étendu de relations et de notre expérience en FMC en hépatogastroentérologie en Afrique Francophone :

http://www.refer.mg/general/agence.htm

\section{L'OMS}

Pourrait également être intéressée par notre projet dynamique et par ses retombées en santé publique en Afrique et nous aider à améliorer notre dimension transnationale. Notre organisation participe à une amélioration de la santé à travers des domaines tels que la nutrition, l'hépatologie, la gastroentérologie, la parasitologie, l'épidémiologie, la cancérologie, l'imagerie, la chirurgie digestive et les sciences paramédicales :

http:/www.who.int/fr/

\section{L'Union africaine}

Elle pourrait nous attribuer un statut d'observateur auprès de ses instances :

http://www.au.int/fr/ 


\section{Les organismes privés}

Il ne faut pas minimiser l'importance des collaborations avec des organismes tels que :

- La Croix Rouge et le croissant rouge

- Des fondations

- Des organisations caritatives

- Des ONG

\section{Mieux se faire connaître}

Un site vient d'être lancé dans cette optique de faire connaître à chacun notre Association, ses buts, son organigramme et ses activités.

Un contact formel vient d'être établi avec le Président de la WGE et une page d'informations sera publiée dans le $J A H G$. http://www.worldgastroenterology.org/wgn.html

Les éditeurs responsables des journaux ou revues de nos sociétés membres pourraient nous ouvrir leurs colonnes afin d'informer et de permettre aux confrères africains de publier leurs travaux.

\section{Assurer une structure permanente de fonctionnement}

Pour permettre d'atteindre ces objectifs, le secrétariat général devrait disposer d'un secrétariat, structure permanente administrative destinée à assurer un meilleur fonctionnement au quotidien de notre association.

Par ailleurs cette structure administrative - en liaison avec le conseil d'administration et le comité scientifique - devrait assurer la préparation, la gestion et la bonne tenue des réunions du bureau et des congrès. 